\title{
ELECTRICAL CHARACTERIZATION OF AN ATMOSPHERIC PRESSURE PLASMA JET
}

\author{
S. K. KC, S. Sharma, R. Shrestha and D. P. Subedi
}

Journal of Nepal Physical Society

Volume 5, Issue 1, October 2019

ISSN: $2392-473 X$

Editors:

Dr. Vinaya Kumar Jha

Dr. Binod Adhikari

Dr. Kapil Adhikari

JNPS, 5 (1), 85-90 (2019)

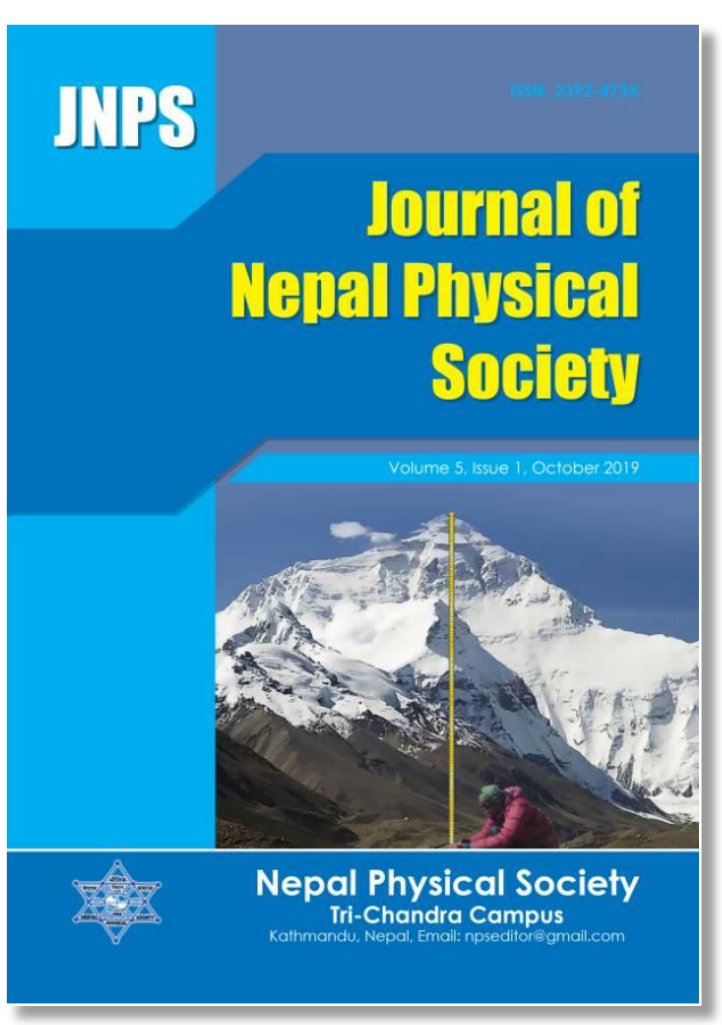

Published by:

Nepal Physical Society

P.O. Box: 2934

Tri-Chandra Campus

Kathmandu, Nepal

Email:npseditor@gmail.com 


\title{
ELECTRICAL CHARACTERIZATION OF AN ATMOSPHERIC PRESSURE PLASMA JET
}

\author{
S. K. KC ${ }^{*}$, S. Sharma ${ }^{2}$, R. Shrestha ${ }^{3}$, D. P. Subedi ${ }^{4 *}$ \\ ${ }^{1}$ Department of Physics, St. Xavier's College, Kathmandu, Nepal \\ ${ }^{2}$ Department of Physics, Patan Multiple Campus, Patan Dhoka, Lalitpur, Nepal \\ ${ }^{3}$ Nepal Banepa Polytechnic College of Engineering, Banepa, Nepal \\ ${ }^{4}$ Department of Natural Science, Kathmandu University, Dhulikhel, Nepal \\ "Corresponding Email: sushil.kc@sxc.edu.np/sushil.kc26@gmail.com
}

\begin{abstract}
In this work, a plasma jet has been generated with capillary tube having external diameter $4.0 \mathrm{~mm}$ and thickness $1.0 \mathrm{~mm}$. An Argon has been used as a working gas. The electrical characteristics of this device like instantaneous power, and discharge current have been measured. The effects of applied voltage on the dissipated power of the device have been investigated. The current is measured with the current probe whereas the voltage is measured from the locally fabricated voltage divider having ratio 1201:1. The electron density has been found out using power balance method. In addition, the power consumption during the discharge has also been studied with the help of Lissajous Figures. The calculated power consumption has been compared with other manual as well as I-V plots.
\end{abstract}

Keywords: Discharge current, electron density, power balance method, Lissajous figures, plasma jet.

\section{INTRODUCTION}

The world has witnessed a remarkable progress in the generation, diagnostics and applications of atmospheric pressure non-thermal and low temperature plasma. Atmospheric pressure plasma jet attracts great attention because of their potential applications in various fields such as polymer surface treatment for biomedical applications- plasma biology, healthcare and medicine as well as material processing and nanotechnology and pollution control $[1,2]$. Atmospheric pressure plasma jets (APPJs) offer a chamberless delivery of downstream reaction, ideal for many applications.

In order to use these plasmas in industrial, biomedical and environmental applications effectively, it is necessary to know the plasma parameters such as the electron temperature $\left(T_{e}\right)$ and electron density $\left(n_{e}\right)$. Therefore to determine the electron temperature and electron density of low pressure plasma, electric or Langmuir probe measurement is widely used [3]. However, a probe theory for atmospheric pressure plasma having a large electron-neutral collision frequency has not yet been established. The application of probe measurement to atmospheric pressure plasma is difficult due to the small inter-electrode distance and due to the problem regarding large heat load and high frequency noise. Therefore as an alternative to probe measurement, optical spectroscopy method is widely used to measure the internal plasma parameters $\left(T_{e}\right.$ and $\left.n_{e}\right)$ under atmospheric pressure environment $[4,5]$.

Various portable APPJ devices including jet needle and plasma pencil have been constructed. For the effective operation of these APPJ devices often molecular gases, such as oxygen or methane is added to the inert gases $[6,7]$. Plasma jets generated using different generation techniques are found to exhibit intriguing properties. APPJs has reduced the necessity of usage of vacuum operation. However, the difficulty of sustenance of a glow discharge under these conditions leads to a new set of challenges. Higher voltages are required for gas breakdown at 760 torr, and often arcing occurs between the electrodes $[8,9]$. So, to prevent arcing, study of physical and chemical properties of APPJs is essential. In this paper, an effort has been made to electrically characterize atmospheric pressure cold plasma jet with respect to supply voltage and frequency to understand the dynamic behavior of APPJs. 


\section{EXPERIMENTAL DETAILS}

Plasma jets concerned in this work are generated in a glass capillary tube with an inner diameter of $3.5 \mathrm{~mm}$ and an outer diameter of $4 \mathrm{~mm}$. The electrodes, $1.0 \mathrm{~cm}$ wide, are made of copper foil wrapping the capillary tube and the distance between the inner edges of two electrodes is in the range of 10 to $20 \mathrm{~cm}$. The grounded electrode is on the upstream side, the active electrode is on the downstream side and $0.5 \mathrm{~cm}$ apart from the

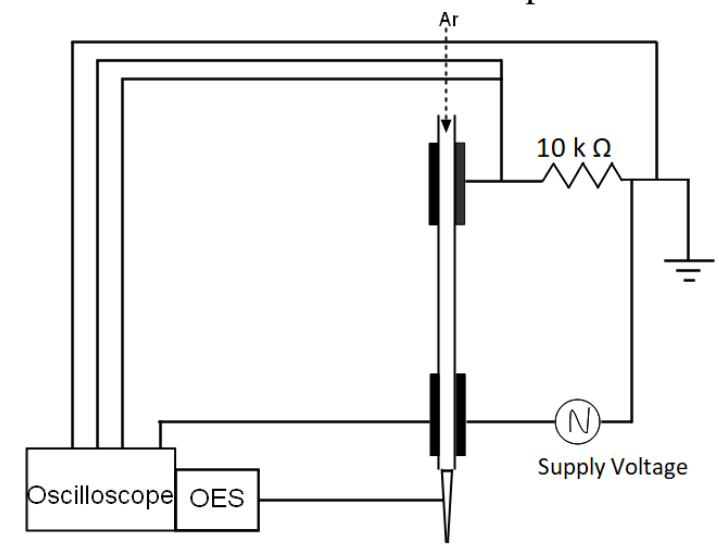

Fig. 1: Schematic of plasma jet device.

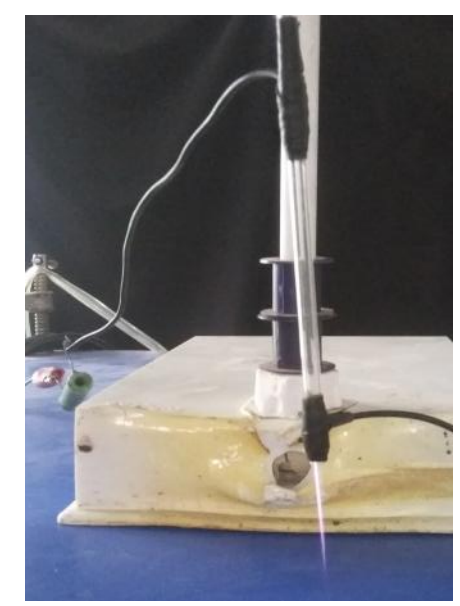

Fig. 2: Photograph of discharge at Plasma lab, Kathmandu University.

tube orifice. Schematic diagram of the present experimental setup of the atmospheric pressure plasma jet apparatus is shown in Figure 1. Argon gas is used as the working gas, and the flow rate is controlled by a volume flow meter. The flow rate of the argon gas was restricted below $5 \mathrm{l} / \mathrm{m}$ so that the flow velocity would not exceed the limit for a laminar argon /oxygen flow. We used a high frequency power supply at a frequency of (25-27) $\mathrm{kHz}$ and a voltage in the range of (0-20) $\mathrm{kV}$ for excitation and sustaining the discharge.
The primary external control parameter for creating the plasma is the applied voltage. It is a sinusoidal voltage and the value is given as an anague value by the power supply. This peak-to-peak value $\left(V_{p p}\right)$ in the experiments was varied in the range (2.5-6.5) $\mathrm{kV}$. The voltage over the plasma was measured with the locally fabricated voltage divider and displayed on the oscilloscope from which it was read out digitally. The sinusoidal voltage was specified by its amplitude.

\section{Calculation of Electron Density From Power Balance Method}

It is the technique commonly used for the estimation of electron density in glow mode of discharge. As the discharge in glow mode is uniformly spread over the whole electrode area, plasma electron density can be obtained from power balance method. The power balance method is based on balance between energy lost by plasma components in various processes and energy gained by plasma from applied power source [10,11,12]. When plasma is in equilibrium, the total energy lost is equal to total energy gained. The energy lost by the plasma per electron-ion pair can be written as

$$
E_{\text {lost }}=V_{p}+E_{e}+E_{\text {coll }} \ldots \ldots \ldots \ldots \text { (1) }
$$

Where $V_{p}$ is plasma potential (kinetic energy gained by each ion falling on the wall sheath), $E_{e}$ is the kinetic energy of electrons lost to the wall and $E_{\text {coll }}$ is the collisional energy lost per electron pair created (includes ionization potential, the excitation energy lost per photon in radiation and elastic collisions with neutrals species). $E_{\text {lost }}$ depends on $T_{e}$, whose value lies between $1 \mathrm{eV}$ and $2 \mathrm{eV}$, under that conditions $E_{\text {lost }}$ can be approximated to $50 \mathrm{eV}$.

The total power absorbed $\left(P_{a b s}\right)$ by capacitively coupled plasma can be written as

$$
P_{a b s}=2 A n_{e} v_{b} e V
$$

Where $\left(2 A n_{e} v_{b} e V\right)$ represents the total discharge current over area $2 \mathrm{~A}$ of the two electrodes, $v_{b}$ is Bohm velocity, $\mathrm{V}$ is voltage and product of e and $\mathrm{V}$ gives $E_{\text {lost }}$. $P_{a v}$ is the average power supply. So, the equation (2) can be written as;

$$
\begin{gathered}
P_{a v}=2 A n_{e} v_{b} E_{\text {lost }} \\
n_{e}=\frac{P_{a v}}{2 A v_{b} E_{l o s t}}
\end{gathered}
$$

This is the required expression used for the calculation of number density. 
Calculation of Power Consumption from Lissajous Figures

A charge-voltage Lissajous figure is a standard method for the electrical diagnostics of DBD discharges as it was initially performed by T.C.Manley on his research titled The Electric Characteristics of Ozonator Discharge [13,14]. In this electrical theory of ozonator, a classical Q-V plot of an ozonator excited by an ac power source has the form of a parallelogram, schematically shown in Figure 3. This is a typical volumedischarge Lissajous figure. From both current and voltage curves, the time-averaged electrical power consumption was obtained from equation;

$$
\begin{array}{r}
\text { Power }=\frac{1}{T} \int_{0}^{T} V(t) I(t) d t \ldots \ldots \ldots \ldots \\
\text { or, Power }=f \int_{0}^{T} V(t) I(t) d t
\end{array}
$$

The $\mathrm{BC}$ and DA sides of the parallelogram are related to the active phases where the slope is equal to the dielectric barrier capacitance $\left.\left(C_{d}\right)\right)$. The area of the Q$\mathrm{V}$ loop is equal to the energy per unit period. This Manley's method has become a standard for the electrical diagnostics of DBDs. However, they are some differences in pulsed volume discharges, e.g., the area represents the energy per pulse, and the $\mathrm{Cd}$ cannot be obtained simply by the slope of the BC side, but rather by the ratio of the maximum charge $\left(Q_{\max }\right)$ to voltage $\left.\left(V_{\max }\right)\right)$ when the applied voltage changes, while the $\mathrm{C}$ total could still be estimated by the slope of the $\mathrm{AB}$ side.

This is the required formula used for the calculation of power using T.C Manley method;

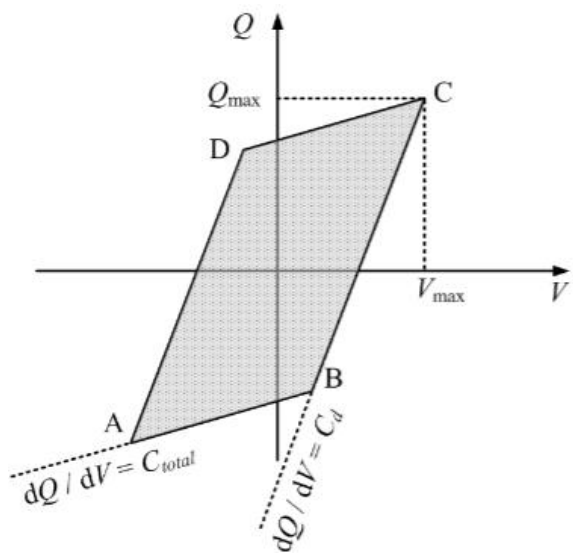

Fig. 3: Schematic representation of Lissajous figures.

Power $=4 V_{0} C_{d} f \frac{C_{d}}{C_{d}+C_{g}}\left(V_{m}-V_{0}\right)$
Where $C_{d}$ is the capacitance of the dielectric material, $C_{g}$ is the capacitance of the gas, $V_{m}$ is the maximum voltage attained after $Q_{\max }, V_{0}$ is the applied voltage, $\mathrm{f}$ is the frequency applied.

The area inside the closed Lissajous curve corresponds to the energy dissipated by the discharge per period [15]. So, for obtaining the dissipated power of the system, the applied voltage and the charge calculated from the integrated value of current is used i.e, $q=i d t$

\section{RESULTS AND DISCUSSION}

Various electrical parameters like current, voltage, power, number density of plasma were measured. Most importantly Lissajous figures have been plotted using T.C. Manley method. These measured values were compared with the previously experimented results and found to be in a good agreement.

\section{Effect of Electrode Separation on Current}

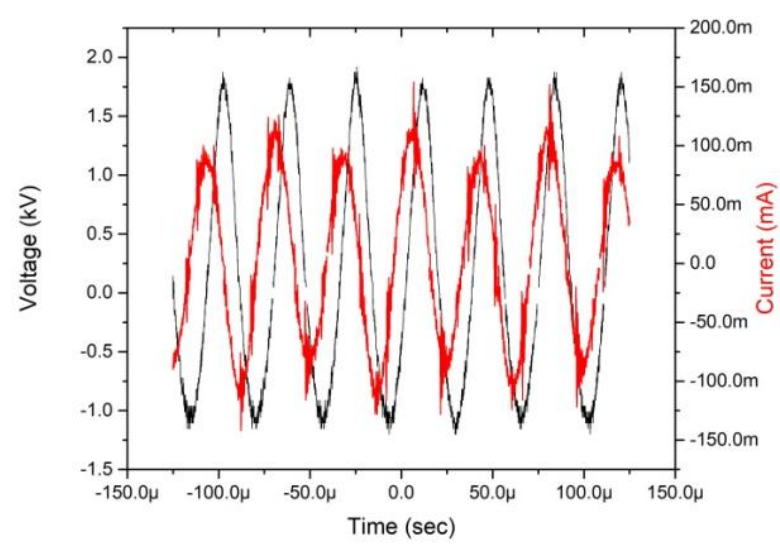

Fig. 4: Current and voltage waveform of APPJ at electrode distance of $10 \mathrm{~cm}$.

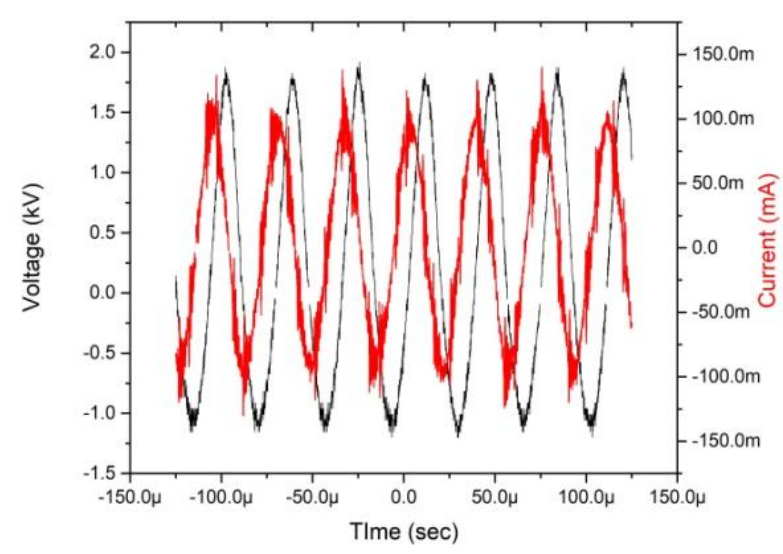

Fig. 5: Current and voltage waveform of APPJ at electrode distance of $13.5 \mathrm{~cm}$. 


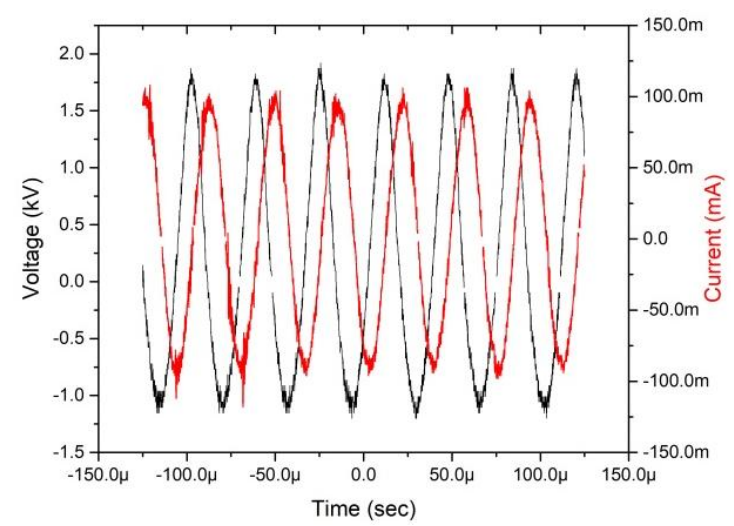

Fig. 6: Current and voltage waveform of APPJ at electrode distance of $16.5 \mathrm{~cm}$.

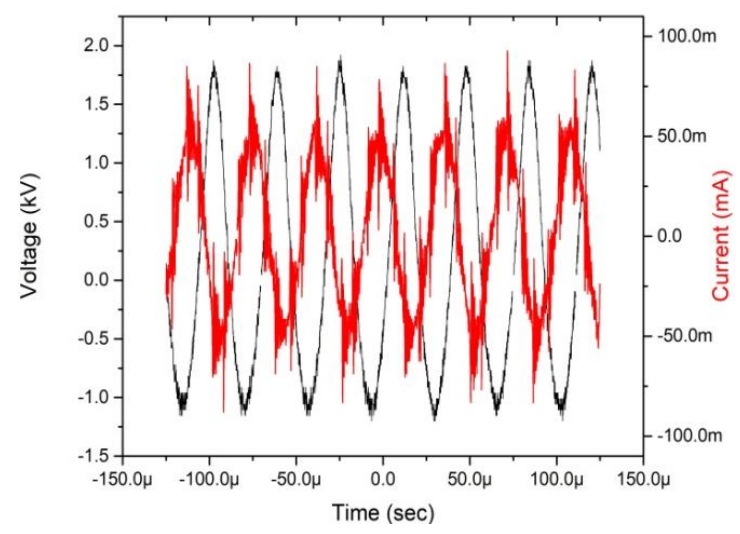

Fig. 7: Current and voltage waveform of APPJ at electrode distance of $20 \mathrm{~cm}$.

The electrons avalanche just after the ionization must have contributed in higher amount of current in the Figure 4 according to the Townsend's ionization theory. Also the electrode separation caused the increment of the mean free path of the collision of the particles and some of the particles do not have sufficient amount of energy to reach another electrode.

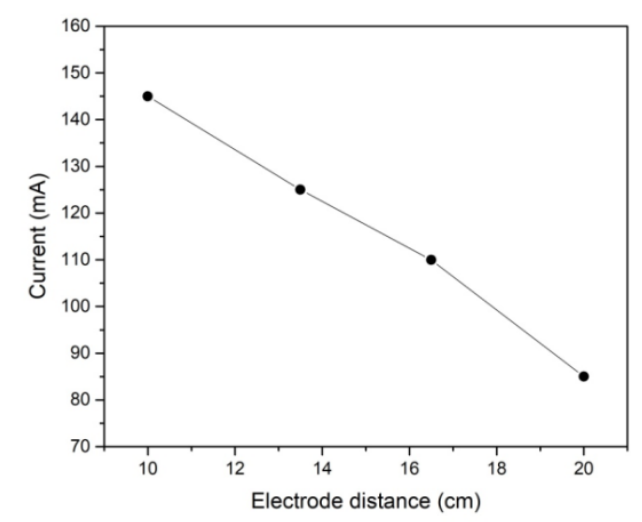

Fig. 8: Discharge current as a function of their inter electrode distance.

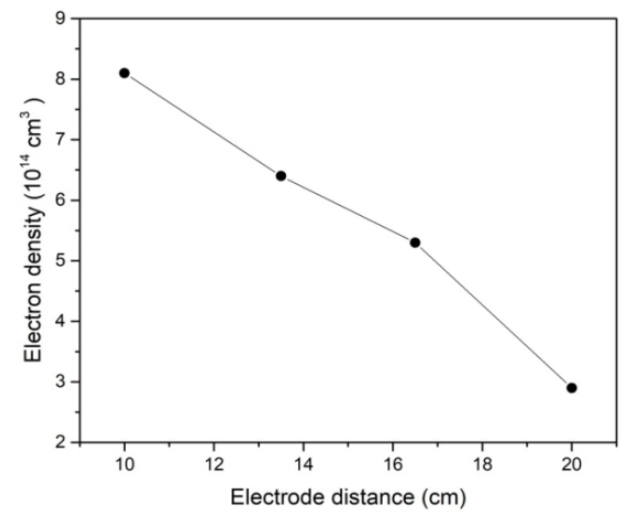

Fig. 9: Variation of electron density with inter electrode distance for applied voltage $(V=4.5 \mathrm{kV})$ and flow rate 2 litre/min.

\section{Effect of applied voltage on current and electron density}

Discharge current, electron density as a function of applied voltage for electrode distance and gas flow rate of liter/min has been studied. Figure 10 shows the linear relationship between the current and voltage and figure 11 shows the increase in electron density with the increase in the applied voltage.

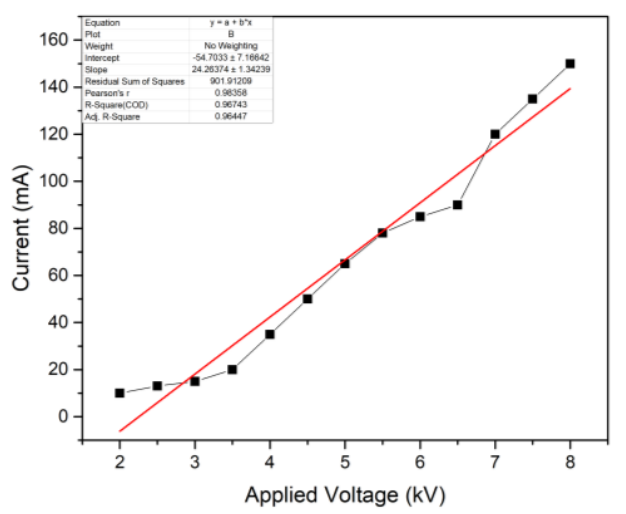

Fig. 10: Discharge current as a function of applied voltage for electrode distance $13.5 \mathrm{~cm}$ and gas flow rate of 2 liter/min.

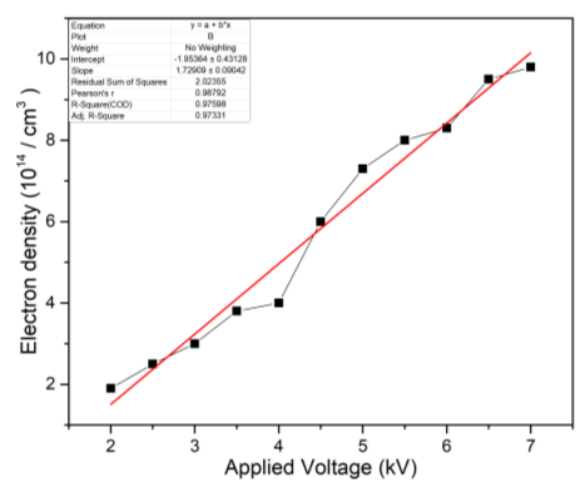

Fig.11: Electron density as a function of applied voltage for electrode distance $13.5 \mathrm{~cm}$ and gas flow rate of 2 litre/min. 


\section{Electrical Power Consumption}

The power calculation in figure 12 was done using $I_{r m s}$ and $V_{r m s}$ values were recorded by digital oscilloscope at different electrode separation. The black line represents power at electrode separation $10 \mathrm{~cm}$, red line for $13.5 \mathrm{~cm}$, green line for $16.5 \mathrm{~cm}$ and blue line for $20 \mathrm{~cm}$ electrode separation. We found out that the power consumption at lower electrode separation was higher compared to higher electrode separation.

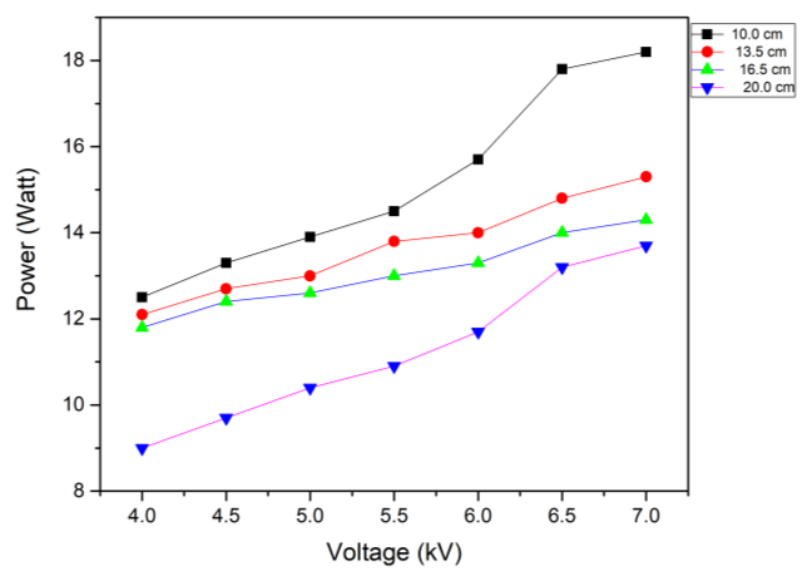

Fig. 12: Variation of power as a function of applied voltage for different values of electrode separation.
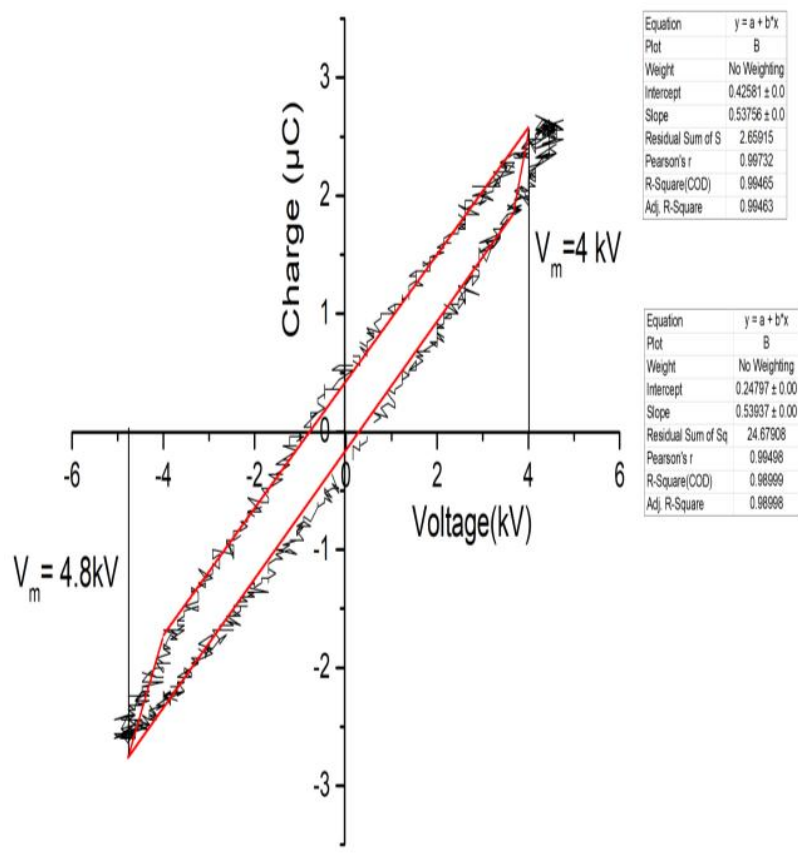

Fig. 13: Sample calculation of power from lissajous figure.

\section{Various Lissajous figures at different electrode separation}

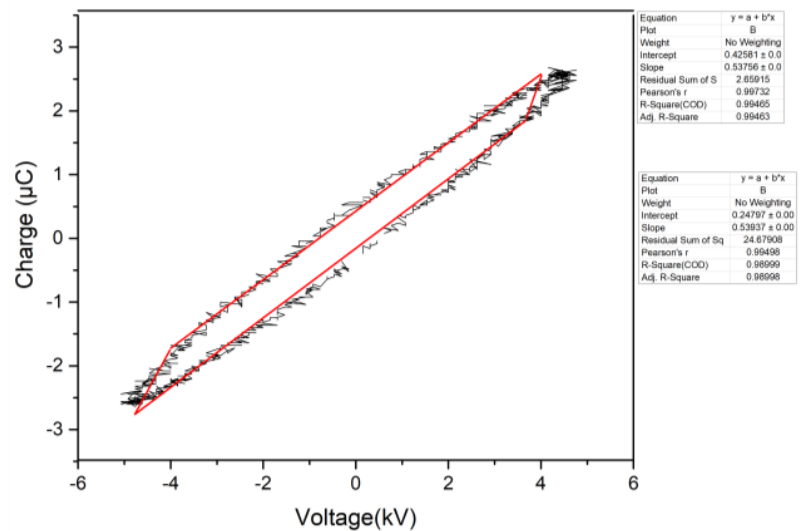

Fig. 14: Lissajous figure at $9 \mathrm{kV}$ peak-peak with electrode separation of $10 \mathrm{~cm}$.

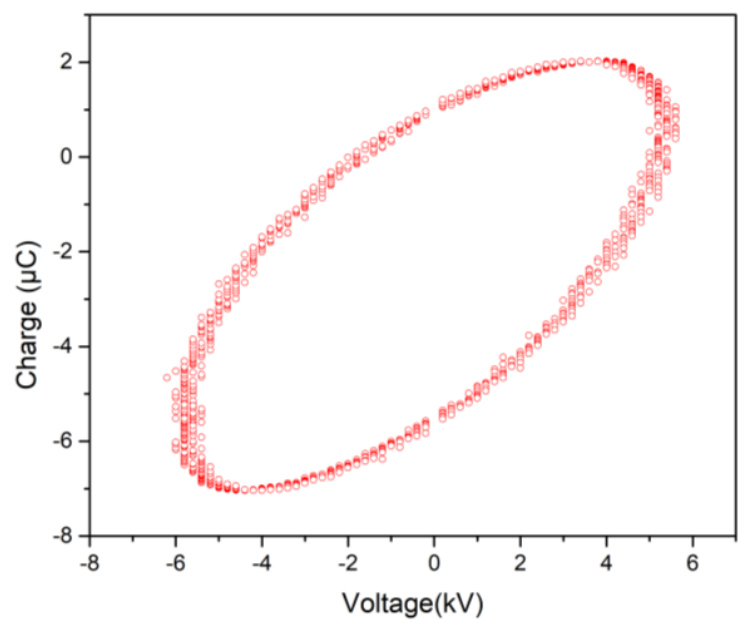

Fig. 15: Lissajous figure at $10 \mathrm{kV}$ peak-peak with electrode separation of $10 \mathrm{~cm}$.

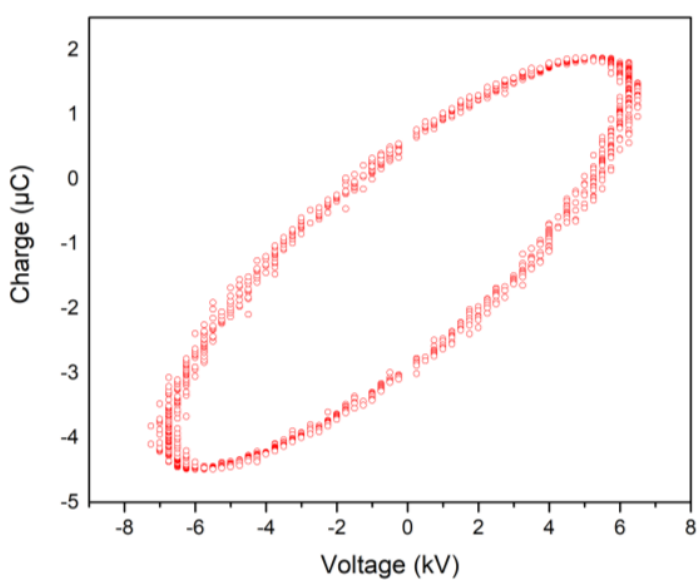

Fig. 16: Lissajous figure at $12 \mathrm{kV}$ peak-peak with electrode separation of $10 \mathrm{~cm}$. 


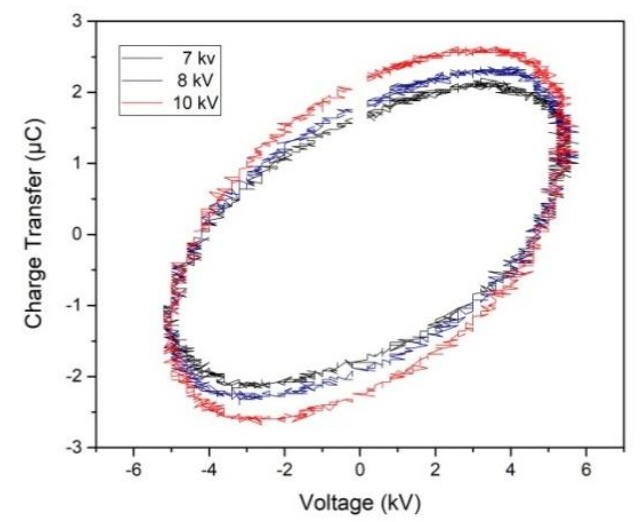

Fig. 17: Lissajous curves for different applied voltages from $7 \mathrm{kV}$ to $10 \mathrm{kV}$ with $10 \mathrm{~cm}$ electrode separation.

\section{CONCLUSION}

In this work, a plasma jet has been generated with glass tube having $4.0 \mathrm{~mm}$ diameter and thickness 1 $\mathrm{mm}$. The electrical parameters of the device like $I_{r m s}$ and $V_{r m s}$ dissipated power, current have been determined. For measuring the time-averaged power of the plasma jet device, a 10 kilo ohm carbon resistor was used. An integrated value of current was used for measuring the charge thereby measuring the dissipated power. The procedure is based on Lissajous curves as an accepted measurement method to determine the consumed power in jet device. The area inside the closed Lissajous curves have been calculated and reported as consumed power per cycle in this work.

The current-voltage and charge-voltage characteristics of the plasma jet have been analyzed for different voltages. The electron density and current have been found to decrease with the increasing electrode distance which corresponds very well with the previous results. For $A C$ voltages in the range from $9 \mathrm{kV}, 10 \mathrm{kV}$ and $12 \mathrm{kV}$, the maximum discharge power obtained was found to increase with the increase in applied voltage.

\section{REFERENCES}

[1] Laroussi, M. \& L U, X.P. Room-temperature atmospheric pressure plasma plume for biomedical applications. Appl. Phys. Lett. 87, 113902 (2005).

[2] Laroussi, M. Nonthermal decontamination of biological media by atmospheric-pressure plasmas: Review, analysis, and prospects. IEEE Trans. Plasma Sci. 30, 1409-1415 (2002).

[3] Eliasson, B. \& Kogelschatz, U. Nonequilibrium volume plasma chemical processing. IEEE Trans. Plasma Sci. 19, 1063-1077 (1991).
[4] Bibinov, N., Dudek, Engemann, J. \& Awakowicz P.Characterization of an atmospheric pressure dc plasma jet. J. Phys. D 40, 736-742 (2007).

[5] Kim, K., Choi, J.D., Hong, Y.C., Kim, G., Noh, E.J., Lee, J.S. \& Yang, S.S. Atmospheric-pressure plasma-jet from micro-nozzle array and its biological effects on living cells for cancer therapy. Applied Physics Letters 98: 073701 (2011).

[6] Joh, H.M., Kim, S.J., Chung, T.H. \& Leem, S.H. Comparison of the characteristics of atmospheric pressure plasma jets using different working gases and applications to plasma-cancer cell interaction. AIP Advances 3: 092128 (2013).

[7] Subedi, D.P., Tyata, R.B., Shrestha, R. \& Wong, C.S. An experimental study of atmospheric pressure dielectric barrier discharge (DBD) in argon. AIP Conference Proceeding 1588: 103-108 (2014).

[8] Shrestha, R., Gurung, J.P., Subedi, D.P. \& Wong, C.S. Atmospheric pressure single electrode argon plasma jet for biomedical applications. International Journal of Emerging Technology and Advanced Engineering 5(11): 193-198 (2015).

[9] Xiong, Q., Nikiforov, A.Y., Gonzalez, M.A., Leys, C. \& Lu, X.P. Characterization of an atmospheric helium plasma jet by relative and absolute optical emission spectroscopy. Plasma Sources Sci. Technol. 22: 015011 (2013).

[10] Jiang, N., Ji, A. \& Cao, Z. Atmospheric pressure plasma jet: Effect of electrode configuration, discharge behavior, and its formation mechanism. Journal of Applied Physics 106: 013308 (2009).

[11] Nastuta, A.V., Pohoata, V. \& Topala, I. Atmospheric pressure plasma jet-living tissue interface: Electrical, optical, and spectral characterization. Journal of Applied Physics 113: 183302 (2013).

[12] Teschke, M., Kedzierski, J., Finantu-Dinu, E.G., Korzec, D. \& Engemann, J. High-speed photographs of a dielectric barrier atmospheric pressure plasma jet. IEEE Transactions on Plasma Science 33, 310-311 (2005).

[13] Park, J. Schutze, A., Jeong, J.Y., Babayan, S.E.,Selwyn, G.S. \& Hicks, R.F. The atmospheric-pressure plasma jet: A review and comparison to other plasma sources. IEEE Trans. Plasma Sci. 26, 1685-1694 (1998).

[14] XU, G.-M., MA, Y. \& G.-J. Zhang. DBD plasma jet in atmospheric pressure argon. IEEE Trans. Plasma Sci. 36, 1352-1353 (2008).

[15] Jeong, J.Y., Babayan, S.E., TU, V.J., Park, J., Hicks, R.F. \& Selwyn, G.S. Etching materials with an atmosphericpressure plasma jet. Plasma Source Sci. Technol. 7, 282-285 (1998). 